\title{
28 Research Square \\ Beliefs and Use of Forefoot Lateral Wedging in \\ Podiatric Practice: A Survey of Podiatrists in Aotearoa New Zealand
}

\section{Aaron Jackson ( $\sim$ Aaron.jackson@aut.ac.nz )}

Auckland University of Technology https://orcid.org/0000-0002-8827-0157

\section{Kelly Sheerin}

Auckland University of Technology

Duncan Reid

Auckland University of Technology

Matthew Carroll

Auckland University of Technology

\section{Research}

Keywords: Podiatry, foot biomechanics, foot orthoses, valgus wedge

Posted Date: October 11th, 2021

DOI: https://doi.org/10.21203/rs.3.rs-944617/v1

License: (c) (i) This work is licensed under a Creative Commons Attribution 4.0 International License.

Read Full License 


\section{Abstract \\ Background}

There is currently limited evidence exploring the beliefs and application of forefoot lateral wedges in clinical practice by podiatrists. The study aimed to understand rationale and beliefs that guided the use of forefoot lateral wedges amongst Aotearoa New Zealand podiatrists.

\section{Methods}

A cross-sectional study of Aotearoa New Zealand podiatrists was conducted between 31st May 2021 and 26th July 2021. Data were collected anonymously using a web-based survey platform. The 30-item survey included questions to elicit participant characteristics, why and when podiatrists used orthotic modifications, what biomechanical assumptions influenced clinical decision making, and how podiatrists fabricated and placed forefoot lateral wedging. The survey findings were reported using descriptive statistics.

\section{Results}

Sixty-five podiatrists completed the survey. Most respondents were trained in Aotearoa New Zealand $(91 \%)$, had over 10 years' experience (70\%), and worked with a mixed case load (60\%). Seventy-seven percent $(77 \%)$ prescribed 0 to 10 pairs of foot orthoses per week, with forefoot lateral wedges used in $44 \%$ of prescriptions. Forefoot lateral wedges were likely to be used in the treatment of peroneal tendon injuries $(70 \%)$ and chronic ankle instability $(64 \%)$. The most common belief being that forefoot lateral wedges increase first metatarsophalangeal joint range of motion (86\%). Forefoot lateral wedges were regularly manufactured from $3 \mathrm{~mm}(74 \%)$, medium density ethylene vinyl acetate (91\%) and positioned from the calcaneo-cuboid joint (53\%) to the sulcus (77\%).

\section{Conclusion}

New Zealand podiatrists frequently use forefoot lateral wedges in clinical practice. Respondents were predominately guided by the underpinning belief that forefoot lateral wedging reduces tissue stress and have their greatest functional impact on first metatarsophalangeal joint range of motion. Forefoot lateral wedges are commonly manufactured from $3 \mathrm{~mm}$ ethylene vinyl acetate, although it is important to be aware that this will translate to differing angles, dependant on width. Further research should be undertaken to explore the influence of forefoot lateral wedges on forefoot function and the effect of wedge length.

\section{Background}


Lateral wedging can be defined as material sloped uniformly to be thicker on the lateral side than the medial side (1). These wedges are commonly added to foot orthoses or shoe insoles in the management of a variety of lower limb pathologies $(1,2)$. Lateral wedging has most commonly been investigated in relation to the biomechanical effects on knee motion, particularly for their role in the management of medial knee osteoarthritis (3). In cohorts with medial knee osteoarthritis, lateral wedging of the foot has been shown to reduce knee adduction moments (1), however, this effect is the subject of controversy (4). With respect to lower limb kinetics and kinematics, lateral wedges have been shown to shift the centre of pressure (COP) laterally $(5-7)$ and increase the external eversion moment of the sub-talar joint $(8,9)$. However, current evidence investigating the effect is limited to a small number of observational studies. Beyond this, limited evidence exists to inform how lateral wedges impact other variables such as kinematics of the forefoot or how the wedge design may affect function.

The extent to which lateral wedges affect foot function is linked to the steepness (gradient) of the slope (10), a variable which is interchangeably described in millimetres (thickness) or degrees (inclination angle) of the wedge. For example, Telfer et al. (11) demonstrated that for every $2^{\circ}$ increase in lateral wedge inclination, peak plantar pressure in the lateral forefoot decreased by $0.74 \%$. Considering the impact of this variable on biomechanical outcomes, clear terminology is important to ensure suitable design decisions are made. Also of relevance to design is the length of wedge. There is limited evidence linking the length of a lateral wedge to a biomechanical influence upon foot function $(7,12)$. For the purposes of this review the following terminology will be applied. A full-length wedge will be defined as a wedge beginning from the most proximal aspect of the insole, under the calcaneus, extending to the distal end of the insole, past the apex of the digits. A forefoot lateral wedge (FLW) will be defined as a wedge beginning from the calcaneo-cuboid joint and extending distally to the sulcus of the foot.

Although evidence exploring clinical beliefs that influence the use of lateral wedges is limited, there are several paradigms of foot function that may be used by clinicians to guide orthotic prescription generally. Frequently cited theories of foot function include Root theory (13), Sagittal Plane Facilitation theory (14), Rotational Equilibrium theory (15) and Tissue Stress theory (16). Root theory, which centres around the concept of sub-talar joint neutral and emphasises the importance of maintaining this position, was one of the foundational theories of foot function. Also taking focus on kinematics of the foot is Sagittal Plane Facilitation theory, which proposes several functions of the foot, including the Windlass mechanism, are essential for efficient transition of weight. Hicks (17) provided the original description of the Windlass mechanism, which linked movement of the arch to first metatarsophalangeal joint (MPJ) kinematics. Despite questions regarding the accuracy of the Windlass mechanism as it was originally described (18), this remains a common consideration when prescribing orthoses (19). Rotational equilibrium attempts to predict function of the foot according to the balance of forces around the sub-talar joint axis. The Tissue Stress theory however is not proposed to be a model to understand foot function, but rather, how to manage it. In this theory the focus is to reduce stress on injured tissues to allow healing.

Previous research has highlighted a difference in prescription habits of Aotearoa New Zealand (NZ) podiatrists compared to those from Australia and the United Kingdom (20). Chapman et al. (20) reported 
that NZ podiatrists prescribe prefabricated orthoses more frequently than both their Australian and British colleagues, and conversely prescribe fewer custom orthoses than both groups. However, this data did not explore the application of full length lateral wedges or FLWs amongst NZ podiatrists.

There is currently limited evidence exploring the beliefs that guide the clinical application of FLWs amongst NZ podiatrists. Therefore, the current study aimed to understand the rationale of NZ podiatrists when they use FLWs, and to identify how lateral wedges are manufactured and applied in clinical practice.

\section{Methods}

This study used a cross-sectional observational design. Data collection was completed anonymously using the web-based survey platform (Qualtrics XM, Provo, UT). The survey was implemented over an eight-week period between 31st May 2021 and 26th July 2021. Participants were recruited via an email invitation sent to all members of the national society, (Podiatry New Zealand), and a research flyer distributed to delegates at the 2021 New Zealand Podiatry Conference. Incentivisation to participate was offered in the form of five prizes of $\$ 100$ shopping vouchers. Consent to participate was obtained through participants completing a yes or no question on the landing page of the survey. If participants did not consent to participate, they were unable to proceed into the survey questions. Ethical approval was obtained from the Auckland University of Technology Ethics Committee (AUTEC, reference number 21/118) prior to commencing data collection.

The survey was piloted through a two stage process. First, six NZ registered podiatrists with an average of 17 years' experience and expertise in biomechanical management and orthoses, completed the survey online and provided written feedback. Following suggested amendments, a focus group with the same practitioners was held to finalise the survey questions. The final survey (Additional File 1) comprised 30 questions, divided into three sections. Participants were able to review responses to previous questions by using the back button at any stage until the survey was completed. The first section related to demographics and asked respondents about their experience, age, geographic region, qualification, type of work and frequency of orthotic prescription. Section two posed questions relating to when and why they used certain modifications, and what biomechanical hypotheses influenced their decisions. Section three contained questions about lateral wedge fabrication and placement. Twenty-three of the survey questions garnered categorical responses $(3-10,13,14,17-19,22-27,29-32)$. Questions 11, 12, 20 and 28 used percentage sliders, questions 15 and 16 were Likert scales. Question 21 asked respondents to indicate what percentage of their FLWs were placed on the sock liner, an orthotic, or on the midsole of the shoe. Participants were required to assign a percentage of their total modifications to each category whilst the online platform created a sum which was required to equal 100.

The first question of Sect. 3 screened respondents to identify those who made their own FLWs. To ensure that those responding to the questions had some experience in wedge fabrication, the final section of the survey was only shown to respondents who reported manufacturing their own FLWs. Any respondents 
who indicated using prefabricated FLWs, skipped the fabrication questions and were taken to the end of the survey. All survey data were reported in accordance with the Checklist for Reporting Results of Internet E-Surveys (Additional file 2) (21).

\section{Data analysis}

All categorical data were described as number ( $\mathrm{n}$ ) and percent (\%). Likert scale data from question 15 were combined for final analysis. 'Very unlikely' and 'unlikely' responses were recorded as 'unlikely'. 'Likely' and 'very likely' responses were recorded as 'likely'. Percentage sliders were represented as a mean (out of 100) and standard deviation (SD).

\section{Results}

\section{Participant characteristics}

In total 88 survey responses were received, representing $25 \%$ of Podiatry New Zealand members and $18 \%$ of registered podiatrists within NZ (based on 481 podiatrists who hold annual practicing certificates). Twenty-three surveys were incomplete and therefore excluded (a 74\% completion rate), leaving 65 which were included for final analysis. Characteristics of the respondents are detailed in Table 1. Most respondents had greater than 10 years' experience $(70 \%, n=46)$, were trained in NZ $(91 \%, n=59)$, reported working with a variety of clinical presentations $(60 \%, n=39)$ and prescribed between 0 and 10 orthotics per week $(77 \%, n=51)$. More than half of the respondents practiced in either Auckland $(34 \%, n=22)$ or Canterbury $(26 \%, n=17)$, and one quarter $(25 \%, n=16)$ held a post-graduate qualification. 
Table 1

Demographic data of participants.

\begin{tabular}{|c|c|}
\hline & $N(\%)$ \\
\hline \multicolumn{2}{|l|}{ Years of experience } \\
\hline $0-3$ years & $9(13.9)$ \\
\hline $4-6$ years & $6(9.2)$ \\
\hline $7-10$ years & $4(6.2)$ \\
\hline $11-15$ years & $15(23.1)$ \\
\hline $16+$ years & $31(47.7)$ \\
\hline \multicolumn{2}{|l|}{ Age } \\
\hline $20-29$ & $10(15.4)$ \\
\hline $30-39$ & $25(38.5)$ \\
\hline $40-49$ & $16(24.6)$ \\
\hline $50-59$ & $9(13.9)$ \\
\hline $60+$ & $5(7.7)$ \\
\hline \multicolumn{2}{|l|}{ Region of practice } \\
\hline Auckland & $22(33.9)$ \\
\hline Waikato & $8(12.3)$ \\
\hline Bay of Plenty & $5(7.7)$ \\
\hline Gisborne & $1(1.5)$ \\
\hline Hawkes Bay & $2(3.1)$ \\
\hline Taranaki & $2(3.1)$ \\
\hline Manawatū-Whanganui & $3(4.6)$ \\
\hline Wellington & $3(4.6)$ \\
\hline Canterbury & $17(26.2)$ \\
\hline Otago & $2(3.1)$ \\
\hline \multicolumn{2}{|l|}{ Highest qualification } \\
\hline Diploma of Podiatry & $9(14.1)$ \\
\hline Bachelors degree & $39(60.9)$ \\
\hline
\end{tabular}




\begin{tabular}{|ll|}
\hline & N (\%) \\
\hline Postgraduate diploma or certificate & $12(18.8)$ \\
\hline Masters degree & $3(4.7)$ \\
\hline Doctoral degree & $1(1.6)$ \\
\hline Undergraduate training institute & \\
\hline AUT University & $34(52.3)$ \\
\hline CIT Wellington & $25(38.5)$ \\
\hline An Australian University & $2(3.1)$ \\
\hline Other & $4(6.2)$ \\
\hline Predominant type of clinical work & \\
\hline General podiatric care & $8(12.3)$ \\
\hline Mixed patient load & $39(60.0)$ \\
\hline Biomechanical/Musculoskeletal & $16(24.6)$ \\
\hline Other & $2(3.1)$ \\
\hline Number of orthoses prescribed per week & \\
\hline $0-10$ & $51(77.3)$ \\
\hline $11-25$ & $13(19.7)$ \\
\hline $26+$ & $2(3.1)$ \\
\hline
\end{tabular}

\section{The use of lateral forefoot wedges}

In relation to the total number of orthotics prescribed, participants reported including a FLW in $44 \%$ (SD $26.76)$ of cases. When asked what modifications they routinely used, 79\% $(n=52)$ reported using FLWs. Only metatarsal domes $(80 \%, n=53)$ were more widely used.

Respondents were asked about their likelihood of using a FLW in the management of various lower limb conditions (Fig. 1). The four conditions for which FLWs were most frequently used included peroneal tendon issues, chronic ankle instability (CAI), plantar heel pain and medial knee osteoarthritis. Conversely, of the list presented to participants first MPJ joint sprain is the diagnosis which least often leads to the use of FLWs.

Figure 2 presents data from the question, "How important do you think it is to consider the following, when using a lateral forefoot wedge". The most important factors for consideration when prescribing 
FLWs were forefoot alignment and rearfoot pronation/supination. These were deemed 'important' by $88 \%$ ( $n=58)$ and $73 \%(n=48)$ of participants respectively.

\section{The biomechanical function of lateral forefoot wedging}

In answer to the question 'How influential do you think each of the following podiatric

theories/paradigms are in your orthotic prescription?, the Tissue Stress theory (81.2/100, SD 20.4) was reported to be the paradigm which most significantly affects orthotic prescription. This was followed by Sagittal Plane Facilitation theory (69.0/100, SD 21.1), Rotational Equilibrium theory $(55.4 / 100$, SD 28.6) and lastly, Root theory (50.2/100, SD 28.4).

The primary reason that NZ podiatrists prescribed lateral forefoot wedging for both running and walking gait, was to increase first MPJ ROM. Whether selected as their main reason for use or not, most podiatrists $(86 \%, n=56)$ agree that this is an expected biomechanical function of lateral forefoot wedging. Figure 3 contrasts the reasons that FLW are prescribed, for both running and walking gait. The majority of respondents $(68 \%, n=45)$ indicated that the biomechanical effect of lateral forefoot wedging is different in running gait, compared to walking gait. However, the three most common reasons for the use of FLWs were the same for both walking and running gait.

\section{Fabrication of lateral forefoot wedges}

Sixty-three percent $(n=42)$ of the respondents in this survey choose to construct their own FLWs. Respondents who reported using prefabricated FLWs most often used wedges manufactured by FootBionics $\AA(52 \%, n=12)$ and Formthotics $\AA(22 \%, n=5)$.

\section{Wedge placement}

Placement of FLWs was considered to have a large impact (81/100, SD 15.9) on their outcome. Seventyseven percent $(n=33)$ of respondents reported not always using the same design of FLWs, with patients symptoms highlighted as the most common reason for adjusting design. The most common FLW design reported began at the calcaneo-cuboid joint (CCJ) and finished at the sulcus of the foot. Beginning this modification at the CCJ was standard for $53 \%(n=23)$ of participants, whilst $40 \%(n=17)$ used the styloid process as their landmark. Less variation was reported regarding the distal border with $77 \%(n=$ 33) agreeing on ending the FLW in the sulcus of the foot. A small number of respondents reported finishing the FLW proximal to the metatarsal heads $(19 \%, n=8)$ or at the end of the insole $(5 \%, n=2)$.

\section{Wedge material}

Respondents considered that material density (82/100, SD 12.4) and thickness (82/100, SD 13.7) were important when constructing FLWs. When asked what materials they have used in the past 12 months, 91\% ( $n=39)$ reported using 'medium density' ethylene vinyl acetate (EVA), whilst 'low density' EVA (51\%, $n=22)$ and felt $(51 \%, n=22)$ were also widely used. The most commonly used FLW material thickness was $3 \mathrm{~mm}$ EVA, 74\% $(n=31)$ of respondents reported having used this in the last 12-month period. Fiftytwo percent $(52 \%, n=22)$ have used $4 \mathrm{~mm}$ EVA, 33\% $(n=14)$ have used $5 \mathrm{~mm}$ EVA and $21 \%(n=9)$ have 
used $6 \mathrm{~mm}$ EVA in this time period. Eight-millimetre EVA has been used by four respondents (10\%) and $10 \mathrm{~mm}$ EVA, by three (7\%). However, no respondents reported using 6,8 or $10 \mathrm{~mm}$ EVA as their primary choice of thickness.

\section{Discussion}

This is the first study to investigate the frequency of application for a range of forefoot orthotic modifications amongst NZ podiatrists. These findings highlight the regular use of FLW within this cohort of NZ based podiatrists, with FLWs reportedly included in almost half of all orthotic prescriptions. This makes them the second most widely applied orthotic modification. This survey identified the majority of respondents prescribed between 0 and 10 orthotics or modified insoles per week. A similar frequency of prescription as that reported by Chapman et al. (20) who found that NZ podiatrists on average prescribed 12 orthoses, per week.

Peroneal tendinopathy and CAI were the diagnoses which most frequently lead to FLW prescription. Both diagnoses have been linked to patients whose COP is laterally deviated $(22,23)$. Therefore, if the widely held assumption revealed in this survey is true, and FLWs do shift the COP medially, this is likely to have a positive impact on these clinical presentations. However, this assumption contrasts current research which suggests that lateral wedges shift the COP laterally (5). There is currently limited evidence investigating this function of FLWs, or any positive outcome effect when managing peroneal tendinopathy. It has been postulated that orthoses have a positive impact on CAl, due to heightened input to mechanoreceptors, although limited work has been completed exploring the role of FLWs in this scenario (24). Kakihana et al. (2) compared full length lateral wedges in people with and without ankle instability and concluded that in those with unstable ankles, the change in sub-talar joint moments is the same as age matched controls. Plantar heel pain was the third most likely diagnosis to be managed with FLW. The in vitro study by Kogler et al. (12) provides the only evidence regarding lateral wedging configurations and their impact on the plantar fascia with data indicating that FLW reduced plantar fascial strain. The limitation of this work was the use of cadaveric limbs.

The Tissue Stress theory was found to be the most influential paradigm that guided orthotic modification and prescription. The Tissue Stress theory works on the premise that orthoses are used to reduce stress being placed on a tissue, to a tolerable level (16). Therefore, based on the study data, the assumption made when using FLWs under the guidance of the tissue stress model is that FLW will positively impact tissue stresses.

This survey has revealed incongruity between the surmised function of FLW and the inferred explanations of podiatric theories of foot function. This is highlighted in two examples related to the application of the Root theory and Sagittal Plane Facilitation theory. Regarding the Root theory, almost a quarter of respondents primarily use FLW to 'balance the foot', and most respondents identified the patient's forefoot position and rearfoot pronation/supination as 'important' when prescribing FLWs. These concepts are key pillars of the Root theory $(13,25)$, despite the Root theory being rated as the least 
influential paradigm by respondents. Regarding the Sagittal Plane Facilitation theory, almost all respondents believed that FLW increased the ROM in the first MPJ, and this was reported as the most common reason for FLW use in both walking and running gait. However, there is very limited evidence exploring the impact of FLWs on first MPJ kinematics. The Sagittal Plane Facilitation theory, ranked by participants as the second most influential paradigm, places a great deal of importance on first MPJ ROM (14). This theory suggests that first MPJ movement to engage the windlass mechanism is essential for efficient forwards transfer of weight. The Windlass mechanism was first discussed by Hicks (17) and relates to the association between MPJ position and the plantar fascia, said to cause the medial arch to rise and the forefoot to supinate. The contradiction in this case is that Hicks (17) described the irresistible supination of the forefoot as the windlass mechanics is engaged, yet over half of our respondents believe that FLWs both shift the COP medially and increase first MPJ ROM. Extrapolation of the original windlass mechanism description would suggest that first MPJ range of motion cannot increase at the same time as the COP shifts medially. However, recent data has shown the plantar fascia to be extensible (18), something that was not considered in the original explanations of the Windlass mechanism. What was previously thought to be a direct link between first MPJ kinematics and medial arch height, appears to be an oversimplification of a complex interaction between the plantar fascia and intrinsic muscles of the foot $(18,26)$. If extensibility of the plantar fascia also impacts the associated forefoot supination, then this could create the possibility of a concurrent medial shift in COP alongside an increase in first MPJ ROM, as participants in the current survey believe is true. However, there is limited evidence to support this supposition and further investigation is required.

Data indicated respondents believed FLW functioned differently in walking gait versus running gait. Respondents also indicated that the biomechanical objectives for FLW prescription were entirely different when managing runners and that running gait enhances the effect of a FLW. Whilst research has indicated biomechanical outcomes derived from orthoses differ between walking and running gait (27), there is limited data supporting functional differences from FLW use between walking and running gait. Given the differing use and the beliefs of clinicians that there are functional differences when using FLW in running versus walking gait, further investigation is warranted.

Full-length lateral wedges have frequently been examined in research, notably for their biomechanical effect in the management of medial knee osteoarthritis (3). However as indicated by survey data, full length wedges made up a small percentage of lateral wedges prescribed by respondents. Evidence contrasting the effect of varying wedge length (full length vs FLW) is limited. Van Gheluwe et al. (7) compared the impact of lateral wedge length on plantar pressure and found FLW increased peak plantar pressure in the lateral forefoot, whereas rearfoot lateral wedges had no effect on forefoot plantar pressure. Further supporting the biomechanical impact of FLW, Kogler et al. (12) demonstrated that wedging under the lateral aspect of the forefoot provided the most significant reduction in strain in the plantar fascia.

The most common material thickness used to manufacture FLWs was $3 \mathrm{~mm}$. However, it is important that the difference between inclination angle and material thickness (in the case of wedges, referring to the 
thickest part) is clearly understood. Three-millimetre $(3 \mathrm{~mm})$ material does not produce a consistent angle as this depends on the width of the modification. Figure 4 displays the relationship between thickness and width, in which the width of a wedge entirely changes the inclination angle if the thickness remains constant. For example, $3 \mathrm{~mm}$ material bevelled to $0 \mathrm{~mm}$ over a width of $60 \mathrm{~mm}$ (a relatively small FLW) produces an inclination angle of $2.9^{\circ}$, whereas that same material bevelled over $100 \mathrm{~mm}$ (a large FLW) produces an angle of $1.7^{\circ}$. This difference means if the same material thickness is used regardless of modification width, patients with larger feet receive wedges with lower inclination angles. This is an important distinction as research has previously indicated that for a range of biomechanical outcomes, a larger inclination angle elicits a larger response (11).

The survey data must be considered in the context of its limitations. Firstly, despite efforts to maximise recruitment, the sample size was lower than anticipated, which may limit the generalisability of the data. Whilst the response rate was low, recent NZ podiatry workforce data indicated only $19 \%$ of NZ podiatrists worked in the area of sports medicine (28). Consequently, the survey may have only been of interest to a relatively small percentage of the NZ podiatry workforce. However, the study was the first to examine practice habits related to FLW. The responses allowed participants to select a range of common presentations, modifications and beliefs that underpin the prescription of FLW. This allowed space for reflection on their practice habits and established a benchmark for current clinical practice in NZ.

\section{Conclusion}

NZ Podiatrists frequently use FLW. These were generally manufactured from $3 \mathrm{~mm}$, medium density EVA and positioned from the $\mathrm{CCJ}$ to the sulcus. The most common rationales for use were to increase first MPJ ROM, shift the COP medially, and balance the foot. These clinical applications however do not align with podiatric theories of foot function reported to be guiding orthotic prescription. Survey data also highlighted inconsistency in the nomenclature used to describe FLW thickness and inclination. Given the incongruity between theory and practice, further research is required to investigate the mechanism of action for FLW.

\section{Abbreviations}

CAl: Chronic ankle instability

CCJ: Calcaneo-cuboid joint

CHERRIES: Checklist for Reporting Results of Internet E-Surveys

COP: Centre of pressure

EVA: Ethylene vinyl acetate

FLW: Forefoot lateral wedge 
MPJ: Metatarsophalangeal joint

NZ: Aotearoa/New Zealand

ROM: Range of motion

\section{Declarations}

\section{Ethics approval}

Ethical approval was granted by the Auckland University of Technology Ethics Committee (AUTEC). Application reference 21/118.

\section{Consent for publication}

Participants were advised in the participant information page at the beginning of the survey that anonymous, non-identifiable data would be published. Only data from those who consented has been included.

\section{Availability of data and material}

Request for further details of the data set and queries relating to data sharing arrangements may be submitted to Aaron Jackson (aaron.jackson@aut.ac.nz). The survey did not obtain consent for participant data to be shared, although the present data are anonymised with all personal identifiers removed.

\section{Competing interests}

Matthew Carroll is an Editorial Board member of the Journal of Foot and Ankle Research. All other coauthors declare no competing interests.

\section{Funding}

No funding supported the study.

\section{Authors' contributions}

AJ, MC, DR and KS conceived the study. AJ undertook the data analysis. AJ and MC the first version of the manuscript. All authors reviewed subsequent draft version and approved the final manuscript.

\section{Acknowledgements}

We thank the podiatrists who aided in the piloting of the survey and all NZ podiatrists who participated in the study. 


\section{References}

1. Arnold JB, Wong DX, Jones RK, Hill CL, Thewlis D. Lateral Wedge Insoles for Reducing Biomechanical Risk Factors for Medial Knee Osteoarthritis Progression: A Systematic Review and Meta-Analysis. Arthritis Care Res. 2016;68(7):936-51.

2. Kakihana W, Torii S, Akai M, Nakazawa K, Fukano M, Naito K. Effect of a lateral wedge on joint moments during gait in subjects with recurrent ankle sprain. Am J Phys Med Rehabil. 2005;84(11):858-64.

3. Zafar AQ, Zamani R, Akrami M. The effectiveness of foot orthoses in the treatment of medial knee osteoarthritis: A systematic review. Gait Posture. 2020;76:238-51.

4. Arnold JB. Lateral wedge insoles for people with medial knee osteoarthritis: One size fits all, some or none? Osteoarthritis Cartilage. 2016;24(2):193-5.

5. Fukuchi CA, Lewinson RT, Worobets JT, Stefanyshyn DJ. Effects of lateral and medial wedged insoles on knee and ankle internal joint moments during walking in healthy men. J Am Podiatr Med Assoc. 2016;106(6):411-8.

6. Jones RK, Zhang M, Laxton P, Findlow AH, Liu A. The biomechanical effects of a new design of lateral wedge insole on the knee and ankle during walking. Hum Mov Sci. 2013;32(4):596-604.

7. Van Gheluwe B, Dananberg HJ. Changes in plantar foot pressure with in-shoe varus or valgus wedging. J Am Podiatr Med Assoc. 2004;94(1):1-11.

8. Tse CTF, Ryan MB, Hunt MA. Influence of foot posture on immediate biomechanical responses during walking to variable-stiffness supported lateral wedge insole designs. Gait Posture. 2020;81:21-6.

9. Erhart JC, Mündermann A, Mündermann L, Andriacchi TP. Predicting changes in knee adduction moment due to load-altering interventions from pressure distribution at the foot in healthy subjects. $J$ Biomech. 2008;41(14):2989-94.

10. Butler RJ, Barrios JA, Royer T, Davis IS. Effect of laterally wedged foot orthoses on rearfoot and hip mechanics in patients with medial knee osteoarthritis. Prosthet Orthot Int. 2009;33(2):107-16.

11. Telfer S, Abbott M, Steultjens M, Rafferty D, Woodburn J. Dose-response effects of customised foot orthoses on lower limb muscle activity and plantar pressures in pronated foot type. Gait Posture. 2013;38(3):443-9.

12. Kogler GF, Veer FB, Solomonidis SE, Paul JP. The influence of medial and lateral placement of orthotic wedges on loading of the plantar aponeurosis: An in vitro study. J Bone Joint Surg Am. 1999;81(10):1403-13.

13. Root ML, Orien WP, Weed JH. Normal and abnormal function of the foot. Los Angeles: Clinical Biomechanics Corporation; 1977.

14. Dananberg HJ. Functional hallux limitus and its relationship to gait efficiency. J Am Podiatr Med Assoc. 1986;76(11):648-52. 
15. Kirby KA. Methods for determination of positional variations in the subtalar joint axis. J Am Podiatr Med Assoc. 1987;77(5):228-34.

16. McPoil TG, Hunt GC. Evaluation and management of foot and ankle disorders: Present problems and future directions. J Orthop Sports Phys Ther. 1995;21(6):381-8.

17. Hicks JH. The mechanics of the foot, part II: the plantar aponeurosis and the arch. J Anat. 1954;88:25-30.

18. Welte L, Kelly LA, Kessler SE, Lieberman DE, D'Andrea SE, Lichtwark GA, et al. The extensibility of the plantar fascia influences the windlass mechanism during human running. Proc Biol Sci. 2021;288(1943):20202095.

19. Chan F, Bowlby MA, Christensen JC. Medial Column Biomechanics: Nonsurgical and Surgical Implications. Clin Podiatr Med Surg. 2020;37(1):39-51.

20. Chapman LS, Redmond AC, Landorf KB, Rome K, Keenan AM, Waxman R, et al. A survey of foot orthoses prescription habits amongst podiatrists in the UK, Australia and New Zealand. J Foot Ankle Res. 2018;11:64.

21. Eysenbach G. Improving the quality of Web surveys: the Checklist for Reporting Results of Internet ESurveys (CHERRIES). J Med Internet Res. 2004;6(3):e34.

22. Moisan G, Descarreaux M, Cantin V. Effects of chronic ankle instability on kinetics, kinematics and muscle activity during walking and running: A systematic review. Gait Posture. 2017;52:381-99.

23. Bavdek R, Zdolsek A, Strojnik V, Dolenec A. Peroneal muscle activity during different types of walking. J Foot Ankle Res. 2018;11:50.

24. Richie DH, Jr. Effects of foot orthoses on patients with chronic ankle instability. J Am Podiatr Med Assoc. 2007;97(1):19-30.

25. Root ML, Orien WP, Weed JH, Hughes R. Biomechanical examination of the foot. Los Angeles: Clinical Biomechanics Corporation; 1971.

26. Sichting F, Ebrecht $F$. The rise of the longitudinal arch when sitting, standing, and walking: Contributions of the windlass mechanism. PLoS One. 2021;16(4):e0249965.

27. Eng JJ, Pierrynowski MR. The effect of soft foot orthotics on three-dimensional lower-limb kinematics during walking and running. Phys Ther. 1994;74(9):836-44.

28. Carroll M, Jepson H, Molyneux P, Brenton-Rule A. The New Zealand podiatry profession - a workforce in crisis? J Foot Ankle Res. 2020;13(1):62.

\section{Figures}




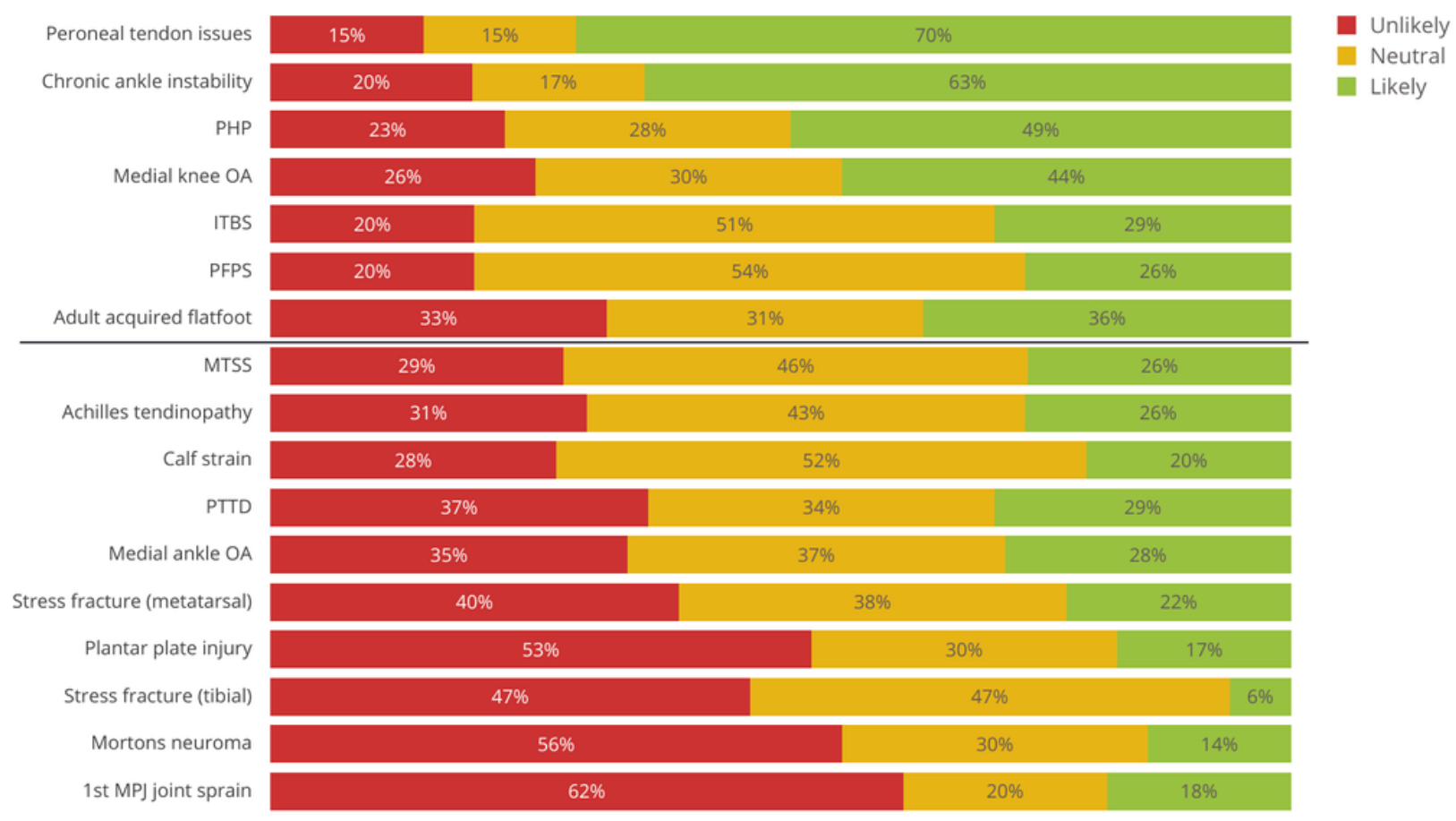

\section{Figure 1}

Likelihood of FLW prescription, based on common lower limb diagnoses. Data above the line indicating the conditions where LWFs were more likely to be used. Data below the line indicates conditions where FLWs were more unlikely to be used 


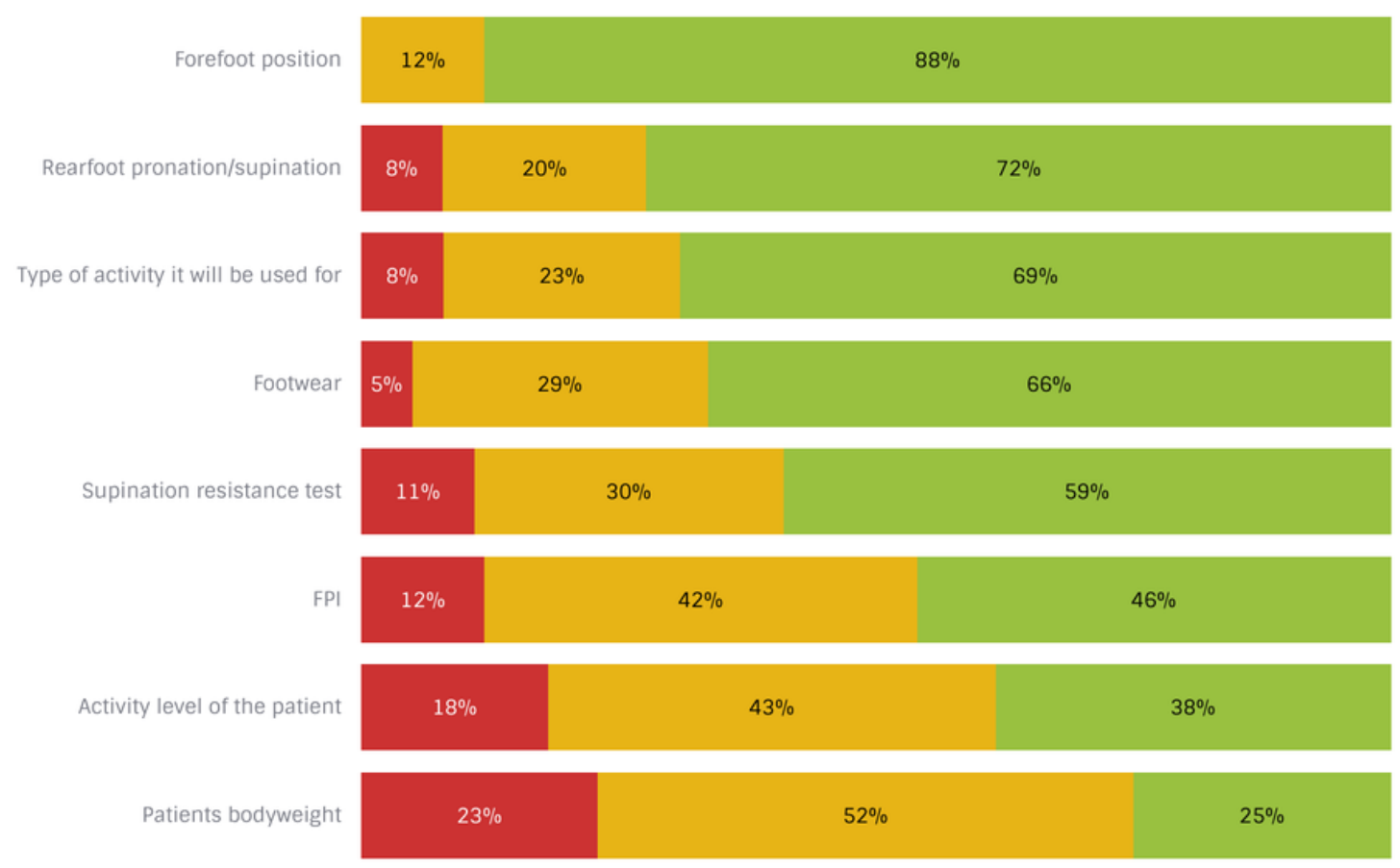

\section{Figure 2}

\section{Importance of various considerations when prescribing lateral forefoot wedging.}

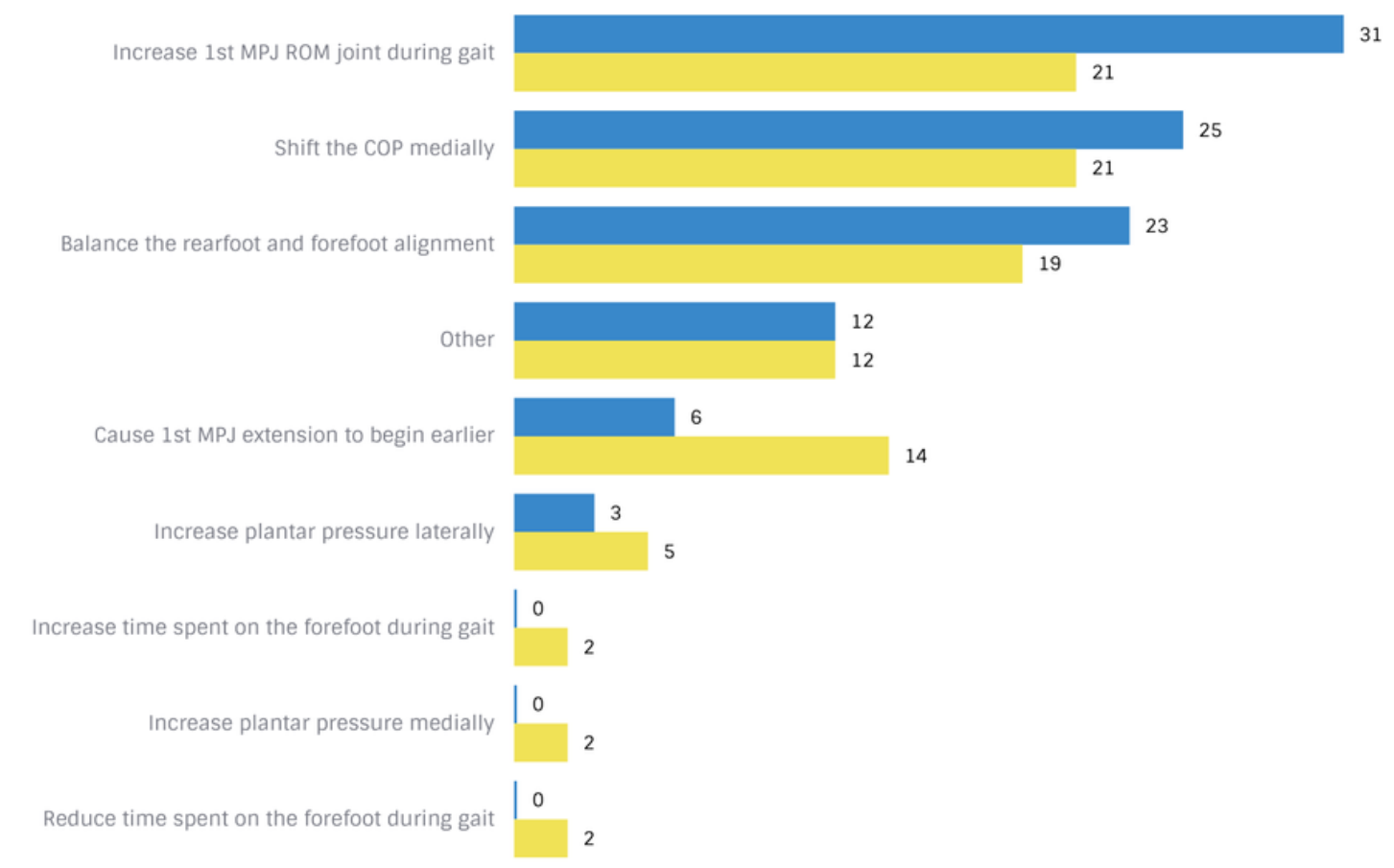




\section{Figure 3}

The most common reasons respondents would prescribe FLWs for both running and walking gait (\%).

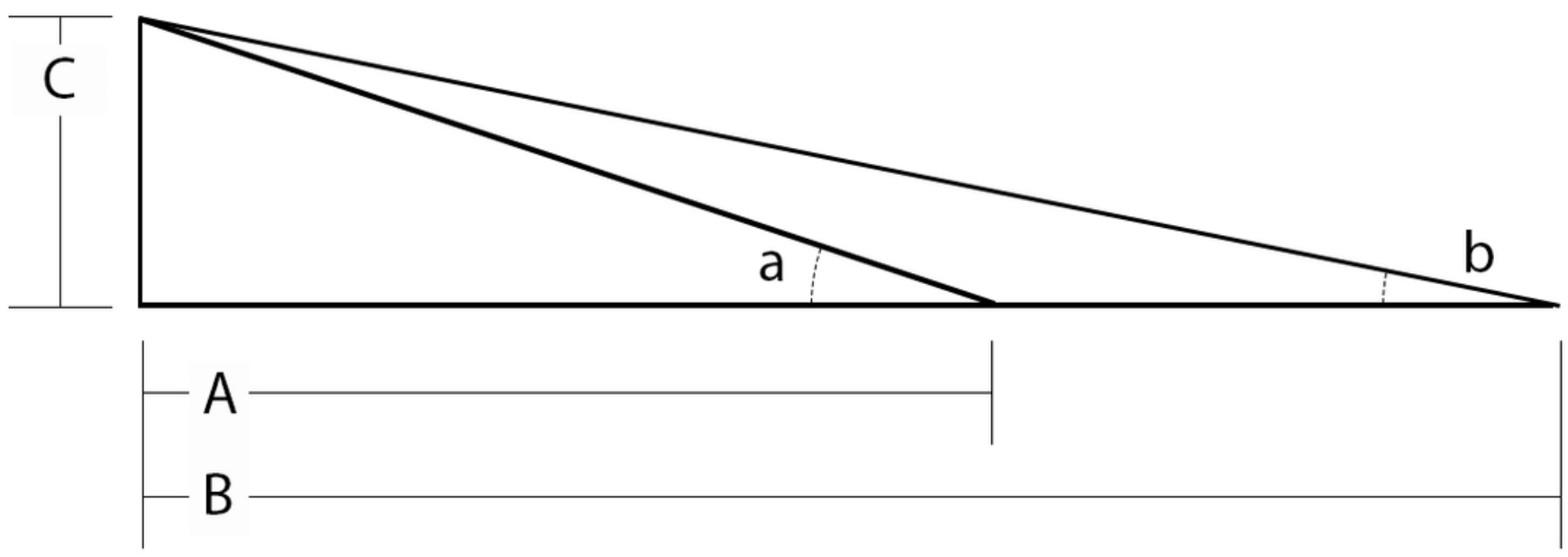

\section{Figure 4}

The relationship between material thickness and width means that at a consistent material thickness (C) the inclination angle (b) will be smaller than (a) due to the modification being wider $(B>A)$.

\section{Supplementary Files}

This is a list of supplementary files associated with this preprint. Click to download.

- Additionalfile1Survey.docx

- Additionalfile2CHERRIES.docx 